\title{
Top food sources of energy and nutrients-to-limit among Latin Americans: Latin American Study of Nutrition and Health Study (ELANS) 2014-2015
}

\author{
Regina Mara Fisberg $^{1}$, Ana Carolina Leme ${ }^{1}$, Aline Veroneze de Mello ${ }^{1}$, Cristiane Salles ${ }^{1}$, \\ Angela Arroyo ${ }^{1,2}$, Georgina Gómez ${ }^{3}$, Irina Kovalskys ${ }^{4}$, Rossina Gabriella Pareja Torres ${ }^{5}$, \\ Martha Cecilia Yépez García ${ }^{6}$, Lilia Yadira Cortés Sanabria ${ }^{7}$, Marianella Herrera-Cuenca ${ }^{8}$, \\ Attilio Rigotti ${ }^{9}$, Mauro Fisberg ${ }^{10}$ and ELANS Group ELANS Group ${ }^{10}$ \\ ${ }^{1}$ Department of Nutrition, School of Public Health, University of São Paulo, São Paulo, Brazil, \\ ${ }^{2}$ Escuela de Nutricion Y Dietética, Universidad de Valparaíso, Valparaíso, Chile, \\ ${ }^{3}$ Departamento de Bioquímica, Escuela de Medicina, Universidad de Costa Rica, San José, Costa Rica, \\ ${ }^{4}$ Committee of Nutrition and Wellbeing, International Life Science Institute, Buenos Aires, Argentina, \\ ${ }^{5}$ Instituto de Investigación Nutricional, La Molina, Lima, Peru, \\ ${ }^{6}$ Colegio de Ciencias de la Salud, Universidad San Francisco de Quito, Quito, Ecuador, \\ ${ }^{7}$ Departamento de Nutrición y Bioquímica, Pontificia Universidad Javeriana, Bogotá, Colombia, \\ ${ }^{8}$ Centro de Estudios del Desarrollo, Universidad Central de Venezuela (CENDES-UCV)/Fundación Bengoa, Caracas, \\ Venezuela, Bolivarian Republic of, \\ ${ }^{9}$ Centro de Nutrición Molecular y Enfermedades Crónicas, Departamento de Nutrición, Diabetes y Metabolismo, \\ Escuela de Medicina, Pontificia Universidad Católica, Santiago, Chile and \\ ${ }^{10}$ Instituto Pensi, Fundação Jose Luiz Egydio Setubal, Sabará Hospital Infantil, São Paulo, Brazil
}

\section{Abstract}

Introduction: Although evidence shows some decrease in

energy intake, consumption of added sugars, solid fat acids (SFA), and sodium are still high among Latin Americans. This study evaluated top food sources contributing to the percentage of energy and nutrients-to-limit among Latin Americans.

Materials and Methods: Latin American Study of Nutrition and Health (ELANS) cross-sectional included 9,218 adults from Argentina, Brazil, Chile, Colombia, Costa Rica, Ecuador, Peru, and Venezuela. 24h-recalls were used and foods were identified via adaptation of "What We Eat in America" system. Food sources of energy and nutrient-to-limit were ranked based on the percentage of intake contribution.

Results: Argentina energy food sources were pizza (11.8\%), and meats (5.7\%); added sugars were sweetened beverages (14.3\%), and quick breads (12.4\%); SFA was pizza $(22.2 \%)$ and meats $(7.8 \%)$; and sodium was pizza $(15.5 \%)$, and soup $(7.6 \%)$. Brazil energy sources were alcoholic beverages $(9.3 \%)$, and pizza (6.9\%); added sugars were sweetened beverages $(14.7 \%)$ and desserts $(14.3 \%)$; SFA and sodium were pizza $(9.0 \%$ and $9.9 \%)$ and sandwiches $(9.4 \%)$. Chile energy sources were pizza $(11.9 \%)$ and grain-based dishes (5.6\%); added sugars were sweet bakeries $(16.6 \%)$ and sweetened beverages $(13.8 \%)$; SFA and sodium were pizza $(19.6 \%$ and $21.2 \%)$ and sandwiches $(7.4 \%$ and $7.7 \%)$. Colombia energy sources were pizza $(6.6 \%)$ and alcoholic beverages $(5.6 \%)$; added sugars were snacks $(15.2 \%)$ and desserts $(12.9 \%)$; SFA were desserts $(9.7 \%)$ and pizza $(7.6 \%)$; and sodium were soups $(11.8 \%)$ and pizza $(10.5 \%)$. Costa Rica energy sources were pizza $(8.7 \%)$ and alcoholic beverages $(6.9 \%)$, added sugars were sweetened beverages $(13.0 \%)$ and candy $(10.5 \%)$; SFA was pizza (12.0\%) and Mexican dishes $(8.9 \%)$; and sodium was pizza (13.5\%) and sandwiches $(8.8 \%)$. Ecuador energy sources were grain dishes $(7.7 \%)$ and alcoholic beverages $(6.8 \%)$, added sugars were sweetened beverages $(14.6 \%)$ and desserts (12.6\%), SFA was pizza (8.8\%) and grain-based dishes (7.5\%), and sodium were Asian dishes (10.4\%) and grainbased dishes $(9.2 \%)$. Peru energy sources were grain-based dishes $(8.9 \%)$ and alcoholic beverages $(8.2 \%)$, added sugars were yogurts $(18.6 \%)$ and sweetened beverages (14.3\%), SFA was pizza $(8.6 \%)$ and sandwiches $(8.1 \%)$, and sodium were grain-based dishes $(17.2 \%)$ and cooked grains (14.9\%). Venezuela energy sources were grain-based dishes $(6.9 \%)$ and alcoholic beverages $(6.1 \%)$, added sugars were sweetened beverages $(13.5 \%)$ and desserts $(11.3 \%)$, SFA were grain-based dishes $(11.3 \%)$ and meats $(7.7 \%)$, and sodium were sandwiches $(9.0 \%)$ and grain-based dishes $(7.9 \%)$.

Discussion: Awareness of food sources is critical for designing strategies to help Latin Americans meet nutrient recommendations within energy needs.

\section{Conflict of Interest}

No Conflict of Interest 